\title{
Weibull-Bayesian Estimation Based on Maximum Ranked Set Sampling with Unequal Samples
}

\author{
B. S. Biradar1, B. K. Shivanna ${ }^{2 *}$ \\ ${ }^{1}$ Department of Studies in Statistics, University of Mysore, Mysuru, India \\ ${ }^{2}$ Department of Statistics, Maharani's Science College for Women, Mysuru, India \\ Email:*shivustats@gmail.com
}

How to cite this paper: Biradar, B.S. and Shivanna, B.K. (2016) Weibull-Bayesian Estimation Based on Maximum Ranked Set Sampling with Unequal Samples. Open Journal of Statistics, 6, 1028-1036. http://dx.doi.org/10.4236/ojs.2016.66083

Received: September 20, 2016 Accepted: November 26, 2016 Published: December 2, 2016

Copyright $\odot 2016$ by authors and Scientific Research Publishing Inc. This work is licensed under the Creative Commons Attribution International License (CC BY 4.0).

http://creativecommons.org/licenses/by/4.0/

\begin{abstract}
A modification of ranked set sampling (RSS) called maximum ranked set sampling with unequal sample (MRSSU) is considered for the Bayesian estimation of scale parameter $\alpha$ of the Weibull distribution. Under this method, we use Linex loss function, conjugate and Jeffreys prior distributions to derive the Bayesian estimate of $\alpha$. In order to measure the efficiency of the obtained Bayesian estimates with respect to the Bayesian estimates of simple random sampling (SRS), we compute the bias, mean squared error (MSE) and asymptotic relative efficiency of the obtained Bayesian estimates using simulation. It is shown that the proposed estimates are found to be more efficient than the corresponding one based on SRS.
\end{abstract}

\section{Keywords}

Bayesian Estimation, Loss Function, MRSSU, SRS, RSS

\section{Introduction}

In certain practical problems, actual measurements of a variable interest are costly or time-consuming, but the ranking items according to the variable is relatively easy without actual measurement. Under such circumstances McIntyre [1] proposed a sampling scheme called ranked-set sampling (RSS) which can be employed to gain more information than simple random sampling (SRS), while keeping the cost of, or the time constraint on, the sampling about the same. In RSS; one first draws $m^{2}$ units at random from the population and partition them into $m$ sets of $m$ units. The $m$ units in each set are ranked without making, an actual measurement. The first set of $m$ units are ranked and the smallest is selected for actual quantification. From the second set of $m$ units, the unit ranked and the second smallest is measured, and so on. This method of selection continues until the unit ranked largest is measured from the $m$-th set. If a 
large sample is required, then the procedure can be repeated $r$ times to obtain a sample of size $n=r m$. These chosen elements are called ranked set sampling. The mathematical support and statistical theory was provided by Takahasi and Wakimoto [2]. Dell and Clutter [3] studied theoretical aspects of this technique on the assumption of perfect and imperfect judgment ranking. Shaibu and Muttlak [4] used median and extreme ranked set sampling method for estimating the parameters of normal, exponential and gamma distributions. Al-Omari et al. [5] Used extreme ranked set sampling method to find the estimates of the population mean. Islam et al. [6] Obtained the modified maximum likelihood estimator of location and scale parameters depend on selected ranked set sampling for normal distribution. Ibrahim and Syam [7] used stratified median ranked set sampling method for estimating the population mean.

Some research works have investigated ranked set sampling from a Bayesian point of view. Varian [8] and Zellner [9] introduced Bayesian estimation by using asymmetric loss functions. Al-Saleh and Muttlak [10] obtained the Bayesian estimates of the exponential distribution. Ahmed [11] obtained the Bayesian estimators of log-normal distribution based on RSS and SRS using Bayes risk. Sadek et al. [12], and Sadek and Alharbi [13] used the asymmetric loss function to obtain the Bayesian estimate of the exponential and Weibull distributions respectively, based on SRS and RSS. Al-Hadhrami and Al-Omari [14] showed that the Bayesian estimation of the mean of normal distribution based on moving extreme ranked set sampling (MERSS) is more efficient than SRS. Hassan [15] obtained the maximum likelihood estimator and Bayesian estimates of shape and scale parameters of the exponentiated exponential distribution based on SRS and RSS. For more research work on Bayesian one may refer to Mohammadi and Pazira [16], Ghafoori et al. [17], Said Ali Al-Hadhrami and Amer Ibrahim Al-Omari [18], Mohie El-Din et al. [19].

In this paper, we derive the Bayesian estimates of the Weibull scale parameter $\alpha$ based on gamma and Jeffreys prior distributions by MRSSU method proposed by Biradar and Santosha [20]. In Section 2, the preliminaries are discussed. The Bayesian estimates under SEL and LINEX loss functions of the parameter of Weibull distribution using SRS and MRSSU are presented in Section 3. Simulation results and Conclusions are presented in Section 4 and 5 respectively.

\section{Preliminaries}

Let $X_{1}, X_{2}, \cdots, X_{m}$ be a sequence of independent and identically distributed (iid) random variables from a Weibull distribution with probability density function (pdf)

$$
f(x)=\alpha \beta x^{\beta-1} \mathrm{e}^{-\alpha x^{\beta}}, x \geq 0, \alpha>0, \beta>0
$$

And cumulative distribution function (cdf)

$$
F(x, \alpha, \beta)=1-\mathrm{e}^{-\alpha x^{\beta}}, x \geq 0, \alpha>0 .
$$

where $\alpha$ is the scale parameter and $\beta$ is shape parameter.

In order to derive, and to measure the performance of an estimator we use squared error, loss function (SEL) (see, Berger [21]) and Linex loss function. 
The Linex loss function for the parameter $\alpha$ can be expressed as

$$
L(\Delta)=d\left(\mathrm{e}^{c \Delta}-c \Delta-1\right),
$$

where $\Delta=(\hat{\alpha}-\alpha) ; \hat{\alpha}$ is an estimate of $\alpha$ and, $c$ and $d$ are shaped and scale parameters. The sign and magnitude of the shape parameter $c$ indicate that the direction and degree of symmetry, respectively. When the value of $c$ is zero, the Linex loss function is approximately squared error loss, when $c$ is less than zero, the Linex loss function gives more weight to under-estimation against over-estimation, and it is reversed when $c$ value is greater than zero. The conjugate prior for $\alpha, \operatorname{Gamma}(a, b)$ is considered, whose pdf is given by

$$
\pi(\alpha)=\frac{b^{\alpha} \alpha^{a-1}}{\Gamma(\alpha)} \mathrm{e}^{-b \alpha}, \quad 0<\alpha<\infty,
$$

where $a, b>0$. If $a=b=0$, then $\pi(\alpha)$ becomes the Jeffreys prior.

\section{Bayesian Estimates}

In this section, we derive the Bayes estimates of the Weibull parameter $\alpha$ based on simple random sampling and maximum ranked set sampling with unequal samples by assuming that the shape parameter $\beta$ is known. In each case, we use both conjugate and non-informative prior for the scale parameter $\alpha$. Also, we use the symmetric loss function (squared error loss) and asymmetric loss function (Linear-exponential, Linex) to derive the corresponding Bayesian estimates. And we denote $k(\alpha \mid \underline{X})$ and $k(\alpha \mid \underline{Y})$ as posterior densities of $\alpha$, given $\operatorname{SRS}(\underline{X})$ and $\operatorname{RSS}(\underline{Y})$ respectively.

\subsection{Bayesian Estimation Based on SRS}

Let $X_{1}, X_{2}, \cdots, X_{m}$ be a sequence of iid random variables, has the Weibull distribution with parameters $(\alpha, \beta)$ and $\pi(\alpha)$ be the conjugate prior. In this case, the posterior density based on SRS is given by

$$
k(\alpha \mid \underline{x})=\frac{\alpha^{m+a-1} \mathrm{e}^{-\alpha\left(b+\sum_{i=1}^{m} x_{i}^{\beta}\right)}\left(b+\sum_{i=1}^{m} x_{i}^{\beta}\right)^{(m+a)}}{\Gamma(m+a)} .
$$

Hence, the Bayesian estimation of $\alpha$ depend on squared error loss (SEL) is $\theta_{\text {Sel }}^{*}=E(\theta \mid \underline{X})$ because the Bayes estimate with respect to SEL is the posterior mean then

$$
\alpha_{S e l}^{*}(\underline{X})=\int_{0}^{\infty} \alpha k(\alpha \mid \underline{x}) \mathrm{d} \alpha=\frac{m+a}{b+\sum_{i=1}^{m} x_{i}^{\beta}} .
$$

While the Bayesian estimate of $\alpha$ based on Linex loss function is

$$
\alpha_{\text {Lnx }}^{*}=-\frac{1}{c} \ln \left[E\left(\mathrm{e}^{-c \alpha}\right)\right]
$$

where, 


$$
E\left(\mathrm{e}^{-c \alpha}\right)=\frac{\left(b+\sum_{i=1}^{n} x_{i}^{\beta}\right)^{m+a} \int_{0}^{\infty} \mathrm{e}^{-\alpha\left(b+\sum_{i=1}^{n} x_{i}^{\beta}\right)} \alpha^{m+a-1} \mathrm{~d} \alpha}{\Gamma(m+a)}
$$

Then,

$$
\alpha_{\ln x}^{*}(\underline{X})=-\frac{1}{c} \ln \left[\frac{b+\sum_{i=1}^{n} x_{i}^{\beta}}{b+\sum_{i=1}^{n} x_{i}^{\beta}+c}\right]^{(m+a)} .
$$

\subsection{Bayesian Estimation Based on MRSSU}

Assume that the variable of interest $X$ has density function $f(x \mid \alpha)$ and distribution function $F(x \mid \alpha)$ is known. Let $\left\{X_{i 1}, X_{i 2}, \cdots, X_{i i}\right\}, i=1, \cdots, m$ be $m$ sets of random samples from $X$, and they are independent. Denote, $Y_{i}=\operatorname{Max}\left\{X_{i 1}, X_{i 2}, \cdots, X_{i i}\right\}$, $i=1, \cdots, m$. Let $Y_{1}$ is taken from the first set, $Y_{2}$ is taken from the second set and $Y_{m}$ is taken from the last set, then $\left\{Y_{1}, Y_{2}, \cdots, Y_{m}\right\}$ be a one cycle MRSSU from $X$ and all $Y_{i}$ 's are independent. In this study we assume that there is no error in ranking. The density of $Y_{i}$ has the same density as the $i^{t h}$ order statistic (maximum) of an SRS of size $i$ from $f(y, \alpha)$, i.e., $Y_{i}$ has the density

$$
f\left(y_{i} \mid \alpha\right)=i[F(y, \alpha)]^{i-1} f(y, \alpha) .
$$

Let MRSSU be drawn from Weibull distribution, then the density function of $Y_{i}$ is

$$
f\left(y_{i} \mid \alpha\right)=i\left[1-\mathrm{e}^{-\alpha y_{i}^{\beta}}\right]^{i-1} \alpha \beta y_{i}^{\beta-1} \mathrm{e}^{-\alpha y_{i}^{\beta}}=\sum_{q=0}^{i-1} i\left(\begin{array}{c}
i-1 \\
q
\end{array}\right)(-1)^{q} \alpha \beta y_{i}^{\beta-1} \mathrm{e}^{-\alpha y_{i}^{\beta}(q+1)} .
$$

Then the joint density of MRSSU in this case due to independence of $y_{i}$ 's is given by

$$
\begin{aligned}
g(\underline{y} \mid \alpha) & =\prod_{i=1}^{m} f\left(y_{i} \mid \theta\right)=\prod_{i=1}^{m} \sum_{q=0}^{i-1}\left(\begin{array}{c}
i-1 \\
q
\end{array}\right)(-1)^{q} \alpha \beta y_{i}^{\beta-1} \mathrm{e}^{-\alpha y_{i}^{\beta}(q+1)} \\
& =\sum_{k_{1}=0}^{0} \sum_{k_{2}=0}^{1} \cdots \sum_{k_{m}=0}^{m-1}\left[\prod_{i=1}^{m} A_{k}(i)\right] \alpha^{m} \beta^{m} D_{k}(i) \prod_{i=1}^{m} y_{i}^{\beta-1}, \quad y_{i}>0
\end{aligned}
$$

where $A_{k}(i)=i\left(\begin{array}{c}i-1 \\ k_{i}\end{array}\right)(-1)^{k_{i}}$ and $D_{k}(i)=\mathrm{e}^{-\alpha \sum_{i=1}^{m} y_{i}^{\beta}\left(k_{i}+1\right)}$.

Then the posterior density of $\alpha$ is

$$
\begin{aligned}
k(\alpha \mid \underline{y}) & =\frac{\pi(\alpha) g(\underline{y} \mid \alpha)}{\int_{0}^{\infty} \pi(\alpha) g(\underline{y} \mid \alpha) \mathrm{d} \alpha}=\frac{\sum_{k_{1}=0 k_{2}=0}^{0} \sum_{k_{m}=0}^{1} \cdots \sum_{i=1}^{m-1}\left[\prod_{k}^{m}(i)\right] \alpha^{m+a-1} \mathrm{e}^{-\alpha\left(\sum_{i=1}^{m} y_{i}^{\beta}\left(k_{i}+1\right)+b\right)}}{\sum_{k_{1}=0 k_{2}=0}^{0} \sum^{1} \cdots \sum_{k_{m}=0}^{m-1}\left[\prod_{i=1}^{m} A_{k}(i)\right] \int_{0}^{\infty} \alpha^{m+a-1} \mathrm{e}^{-\alpha\left(\sum_{i=1}^{m} y_{i}^{\beta}\left(k_{i}+1\right)+b\right)} \mathrm{d} \alpha} \\
& =\frac{\sum_{k_{1}=0}^{0} \sum_{k_{2}=0}^{1} \cdots \sum_{k_{m}=0}^{m-1}\left[\prod_{i=1}^{m} A_{k}(i)\right] \alpha^{m+a-1} \mathrm{e}^{-\alpha\left(\sum_{i=1}^{m} y_{i}^{\beta}\left(k_{i}+1\right)+b\right)}}{\sum_{k_{1}=0 k_{2}=0}^{0} \sum_{k_{2}}^{1} \cdots \sum_{k_{m}=0}^{m-1}\left[\prod_{i=1}^{m} A_{k}(i)\right] \Gamma(m+a)\left(\sum_{i=1}^{m} y_{i}^{\beta}\left(k_{i}+1\right)+b\right)^{-(m+a)}} .
\end{aligned}
$$


The Bayes estimate of $\alpha$ based on the squared error loss function is

$$
\begin{aligned}
\tilde{\alpha}_{\text {Sel }}(\underline{Y})= & E(\alpha \mid \underline{Y})=\int_{0}^{\infty} \alpha k(\alpha \mid \underline{y}) \mathrm{d} \alpha \\
= & \frac{\sum_{k_{1}=0}^{0} \sum_{k_{2}=0}^{1} \cdots \sum_{k_{m}=0}^{m-1}\left[\prod_{i=1}^{m} A_{k}(i)\right] \int_{0}^{\infty} \alpha^{m+a-1} \mathrm{e}^{-\alpha\left(\sum_{i=1}^{m} y_{i}^{\beta}\left(k_{i}+1\right)+b+c\right)} \mathrm{d} \alpha}{\sum_{k_{1}=0}^{0} \sum_{k_{2}=0}^{1} \ldots \sum_{k_{m}=0}^{m-1}\left[\prod_{i=1}^{m} A_{k}(i)\right] \Gamma(m+a)\left(\sum_{i=1}^{m} y_{i}^{\beta}\left(k_{i}+1\right)+b\right)^{-(m+a)}} \\
& \sum_{k_{1}=0}^{0} \sum_{k_{2}=0}^{1} \ldots \sum_{k_{m}=0}^{m-1}\left[\prod_{i=1}^{m} A_{k}(i)\right](m+a)\left(\sum_{i=1}^{m} y_{i}^{\beta}\left(k_{i}+1\right)+b\right)^{-(m+a+1)} \\
\sum_{k_{1}=0}^{0} \sum_{k_{2}=0}^{1} \ldots \sum_{k_{m}=0}^{m-1}\left[\prod_{i=1}^{m} A_{k}(i)\right]\left(\sum_{i=1}^{m} y_{i}^{\beta}\left(k_{i}+1\right)+b\right)^{-(m+a)} & .
\end{aligned}
$$

Next, in order to derive the Bayesian estimation of $\alpha$ based on LINEX loss function, first we need to compute the posterior expectation of $\mathrm{e}^{-c \alpha}$ from Equation (7) as

$$
E\left(\mathrm{e}^{-c \alpha}\right)=\frac{\sum_{k_{1}=0}^{0} \sum_{k_{2}=0}^{1} \cdots \sum_{k_{m}=0}^{m-1}\left[\prod_{i=1}^{m} A_{k}(i)\right]\left(\sum_{i=1}^{m} y_{i}^{\beta}\left(k_{i}+1\right)+b+c\right)^{-(m+a)}}{\sum_{k_{1}=0}^{0} \sum_{k_{2}=0}^{1} \cdots \sum_{k_{m}=0}^{m-1}\left[\prod_{i=1}^{m} A_{k}(i)\right]\left(\sum_{i=1}^{m} y_{i}^{\beta}\left(k_{i}+1\right)+b\right)^{-(m+a)}} .
$$

Now the Bayesian estimation of $\alpha$ on LINEX is

$$
\tilde{\alpha}_{L n x}=-\frac{1}{c} \ln \left[E\left(\mathrm{e}^{-c \alpha}\right)\right] \text {. }
$$

where $E\left[\mathrm{e}^{-c \alpha}\right]$ is as derived in Equation (9).

\subsection{Bayesian Estimation Based on Non-Informative Prior}

The non-informative prior distribution of the parameter $\alpha$ is obtained from Equation (3) and it is given by $\pi(\alpha) \propto \frac{1}{\alpha}, \alpha>0$. Then, we obtain the Bayesian estimates of $\alpha$ in this case as follows:

1) Simple Random Sample:

$$
\alpha_{\text {Sel }}^{* j}(\underline{X})=\frac{m}{\sum_{i=1}^{m} x_{i}}
$$

and

$$
\alpha_{L n x}^{* j}(\underline{X})=-\frac{1}{c} \ln \left[\frac{\sum_{i=1}^{n} x_{i}^{\beta}}{\sum_{i=1}^{n} x_{i}^{\beta}+c}\right]^{m} .
$$

2) Maximum ranked set sampling with unequal samples:

$$
\tilde{\alpha}_{S e l}^{j}(\underline{Y})=\frac{\sum_{k_{1}=0}^{0} \sum_{k_{2}=0}^{1} \ldots \sum_{k_{m}=0}^{m-1}\left[\prod_{i=1}^{m} A_{k}(i)\right] m\left(\sum_{i=1}^{m} y_{i}^{\beta}\left(k_{i}+1\right)\right)^{-(m+1)}}{\sum_{k_{1}=0}^{0} \sum_{k_{2}=0}^{1} \ldots \sum_{k_{m}=0}^{m-1}\left[\prod_{i=1}^{m} A_{k}(i)\right]\left(\sum_{i=1}^{m} y_{i}^{\beta}\left(k_{i}+1\right)\right)^{-m}} .
$$


and

$$
\tilde{\alpha}_{L n x}^{j}=-\frac{1}{C} \ln \left[\frac{\sum_{k_{1}=0}^{0} \sum_{k_{2}=0}^{1} \ldots \sum_{k_{m}=0}^{m-1}\left[\prod_{i=1}^{m} A_{k}(i)\right]\left(\sum_{i=1}^{m} y_{i}^{\beta}\left(k_{i}+1\right)+c\right)^{-m}}{\sum_{k_{1}=0 k_{2}=0}^{0} \sum_{k_{m}}^{1} \ldots \sum_{k_{m}=0}^{m-1}\left[\prod_{i=1}^{m} A_{k}(i)\right]\left(\sum_{i=1}^{m} y_{i}^{\beta}\left(k_{i}+1\right)\right)^{-m}}\right] .
$$

\section{Simulation Results}

To illustrate the performance of the derived Bayesian estimates of scale parameter $(\alpha)$ of the Weibull distribution with informative and non-informative prior based on SRS and MRSSU, we carry out the Monte Carlo simulations using R-Software version 3.1.1. We compute bias, mean squared error and relative efficiency of the estimators by assuming the shape parameter $\beta(=0.5)$ is known. The numerical results obtained for fixed values of $\alpha,[\alpha=0.5$ and 1] and sample size $m$ [3, 4 and 5] for 1000 runs. The bias of the Bayesian estimates based on SRS and MRSSU are presented in Table 1 and Table 2, and MSE of the Bayesian estimates based on SRS and MRSSU is presented in Table 3 and Table 4.

Table 1. Bias of the Bayesian estimates based on SRS and MRSSU. For $\alpha=0.5$ (when $\beta=0.5$, $a=1, \quad b=0.5$ ).

\begin{tabular}{|c|c|c|c|c|c|c|c|c|c|}
\hline \multirow[b]{3}{*}{$m$} & \multirow{2}{*}{\multicolumn{2}{|c|}{$\begin{array}{c}\operatorname{Bias}\left(\alpha_{S e l}\right) \\
\text { Jeffrey prior }\end{array}$}} & \multicolumn{2}{|c|}{$\operatorname{Bias}\left(\alpha_{S e l}\right)$} & \multicolumn{3}{|c|}{$\operatorname{Bias}\left(\alpha_{L n x}\right)$} & \multicolumn{2}{|c|}{$\operatorname{Bias}\left(\alpha_{L n x}\right)$} \\
\hline & & & Gam & prior & & Jeff & prior & Gam & prior \\
\hline & SRS & MRSSU & SRS & MRSSU & $c$ & SRS & MRSSU & SRS & MRSSU \\
\hline \multirow[t]{2}{*}{3} & 0.2487 & 0.1151 & 0.3420 & 0.1811 & 1 & 0.1379 & 0.0750 & 0.2433 & 0.1401 \\
\hline & & & & & -1 & 0.4071 & 0.1689 & 0.5318 & 0.2335 \\
\hline \multirow[t]{2}{*}{4} & 0.1585 & 0.0636 & 0.2355 & 0.1068 & 1 & 0.0914 & 0.0429 & 0.1733 & 0.0853 \\
\hline & & & & & -1 & 0.2454 & 0.0874 & 0.3207 & 0.1314 \\
\hline \multirow[t]{2}{*}{5} & 0.1255 & 0.0424 & 0.1936 & 0.0731 & 1 & 0.0794 & -0.0538 & 0.1481 & 0.0231 \\
\hline & & & & & -1 & 0.1831 & -0.0064 & 0.2536 & 0.0664 \\
\hline
\end{tabular}

Table 2. Bias of the Bayesian estimates based on SRS and MRSSU. For $\alpha=1$ (when $\beta=0.5$, $a=1, \quad b=0.5$ ).

\begin{tabular}{|c|c|c|c|c|c|c|c|c|c|}
\hline \multirow[b]{3}{*}{$m$} & \multirow{2}{*}{\multicolumn{2}{|c|}{$\begin{array}{c}\operatorname{Bias}\left(\alpha_{\text {Sel }}\right) \\
\text { Jeffrey prior }\end{array}$}} & \multicolumn{2}{|c|}{$\operatorname{Bias}\left(\alpha_{S e l}\right)$} & \multicolumn{3}{|c|}{$\operatorname{Bias}\left(\alpha_{L n x}\right)$} & \multicolumn{2}{|c|}{$\operatorname{Bias}\left(\alpha_{L n X}\right)$} \\
\hline & & & Gam & a prior & & Jeff & prior & Gam & a prior \\
\hline & SRS & MRSSU & SRS & MRSSU & $c$ & SRS & MRSSU & SRS & MRSSU \\
\hline \multirow[t]{2}{*}{3} & 0.4975 & 0.2302 & 0.4778 & 0.2781 & 1 & 0.1369 & 0.0853 & 0.2209 & 0.1484 \\
\hline & & & & & -1 & 0.8876 & 0.4671 & 1.0272 & 0.4803 \\
\hline \multirow[t]{2}{*}{4} & 0.3171 & 0.1271 & 0.3422 & 0.1700 & 1 & 0.0883 & 0.0493 & 0.1626 & 0.0954 \\
\hline & & & & & -1 & 0.7232 & 0.2321 & 0.6499 & 0.2657 \\
\hline \multirow[t]{2}{*}{5} & 0.2510 & 0.0848 & 0.2939 & 0.1190 & 1 & 0.0864 & -0.1250 & 0.1523 & -0.0088 \\
\hline & & & & & -1 & 0.5139 & 0.0508 & 0.5115 & 0.1397 \\
\hline
\end{tabular}


The relative efficiency of the Bayesian estimates based on maximum ranked set sampling with unequal samples with respect to simple random sampling can be defined as follows

$$
e f f_{(S e l)}=\frac{M S E_{S R S}\left(\alpha_{S e l}\right)}{M S E_{M R S S U}\left(\alpha_{S e l}\right)} \quad \text { and } \quad e f f_{(\text {Lnx })}=\frac{M S E_{S R S}\left(\alpha_{\text {Lnx }}\right)}{M S E_{\text {MRSSU }}\left(\alpha_{\text {Lnx }}\right)}
$$

And are presented in Table 5.

Table 3. MSE of the Bayesian estimates based on SRS and MRSSU. For $\alpha=0.5$ (when $\beta=0.5$, $a=1, b=0.5$ ).

\begin{tabular}{|c|c|c|c|c|c|c|c|c|c|}
\hline \multirow[b]{3}{*}{$m$} & \multirow{2}{*}{\multicolumn{2}{|c|}{$\begin{array}{c}\operatorname{MSE}\left(\alpha_{S e l}\right) \\
\text { Jeffrey prior }\end{array}$}} & \multirow{2}{*}{\multicolumn{2}{|c|}{$\begin{array}{c}\operatorname{MSE}\left(\alpha_{S e l}\right) \\
\text { Gamma prior }\end{array}$}} & \multirow[b]{3}{*}{$c$} & \multirow{2}{*}{\multicolumn{2}{|c|}{$\begin{array}{c}\operatorname{MSE}\left(\alpha_{\operatorname{Lnx}}\right) \\
\text { Jeffrey prior }\end{array}$}} & \multirow{2}{*}{\multicolumn{2}{|c|}{$\begin{array}{c}\operatorname{MSE}\left(\alpha_{L n x}\right) \\
\text { Gamma prior }\end{array}$}} \\
\hline & & & & & & & & & \\
\hline & SRS & MRSSU & SRS & MRSSU & & SRS & MRSSU & SRS & MRSSU \\
\hline \multirow[t]{2}{*}{3} & 0.4193 & 0.1147 & 0.3899 & 0.1357 & 1 & 0.1849 & 0.0800 & 0.2179 & 0.0980 \\
\hline & & & & & -1 & 1.0296 & 0.1870 & 1.2535 & 0.2015 \\
\hline \multirow[t]{2}{*}{4} & 0.2850 & 0.0555 & 0.2470 & 0.0651 & 1 & 0.1389 & 0.0450 & 0.1524 & 0.0528 \\
\hline & & & & & -1 & 0.4709 & 0.0712 & 0.4577 & 0.0823 \\
\hline \multirow[t]{2}{*}{5} & 0.1584 & 0.0304 & 0.1696 & 0.0355 & 1 & 0.1004 & 0.0387 & 0.1170 & 0.0505 \\
\hline & & & & & -1 & 0.2593 & 0.0615 & 0.2823 & 0.0542 \\
\hline
\end{tabular}

Table 4. MSE of the Bayesian estimates based on SRS and MRSSU. For $\alpha=1$ (when $\beta=0.5$, $a=1, \quad b=0.5$ ).

\begin{tabular}{|c|c|c|c|c|c|c|c|c|c|}
\hline \multirow[b]{3}{*}{$m$} & \multirow{2}{*}{\multicolumn{2}{|c|}{$\begin{array}{c}\operatorname{MSE}\left(\alpha_{S e l}\right) \\
\text { Jeffrey prior }\end{array}$}} & \multirow{2}{*}{\multicolumn{2}{|c|}{$\begin{array}{c}\operatorname{MSE}\left(\alpha_{S e l}\right) \\
\text { Gamma prior }\end{array}$}} & \multirow[b]{3}{*}{$c$} & \multirow{2}{*}{\multicolumn{2}{|c|}{$\begin{array}{c}\operatorname{MSE}\left(\alpha_{L n x}\right) \\
\text { Jeffrey prior }\end{array}$}} & \multirow{2}{*}{\multicolumn{2}{|c|}{$\begin{array}{c}\operatorname{MSE}\left(\alpha_{L n x}\right) \\
\text { Gamma prior }\end{array}$}} \\
\hline & & & & & & & & & \\
\hline & SRS & MRSSU & SRS & MRSSU & & SRS & MRSSU & SRS & MRSSU \\
\hline \multirow[t]{2}{*}{3} & 1.6772 & 0.4586 & 0.8381 & 0.3874 & 1 & 0.4288 & 0.2397 & 0.3299 & 0.2208 \\
\hline & & & & & -1 & 3.9104 & 1.2337 & 3.8436 & 0.8687 \\
\hline \multirow[t]{2}{*}{4} & 1.1400 & 0.2220 & 0.5929 & 0.2103 & 1 & 0.3550 & 0.1507 & 0.2771 & 0.1453 \\
\hline & & & & & -1 & 3.0419 & 0.3914 & 1.7406 & 0.3360 \\
\hline \multirow[t]{2}{*}{5} & 0.6337 & 0.1215 & 0.4605 & 0.1228 & 1 & 0.2852 & 0.1327 & 0.2464 & 0.1168 \\
\hline & & & & & -1 & 1.6544 & 0.3654 & 1.0991 & 0.2718 \\
\hline
\end{tabular}

Table 5. Relative efficiency when $\alpha=0.5$ and $\alpha=1$.

\begin{tabular}{|c|c|c|c|c|c|c|c|c|c|}
\hline \multirow[b]{2}{*}{$m$} & \multicolumn{2}{|c|}{ 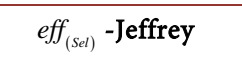 } & \multicolumn{2}{|c|}{ eff $f_{(S e l)}$-Gamma } & \multirow[b]{2}{*}{$c$} & \multicolumn{2}{|c|}{ eff $f_{(\operatorname{Lnx})}$-Jeffrey } & \multicolumn{2}{|c|}{ eff $f_{(\text {Lnx) }}$-Gamma } \\
\hline & $\alpha=0.5$ & $\alpha=1$ & $\alpha=0.5$ & $\alpha=1$ & & $\alpha=0.5$ & $\alpha=1$ & $\alpha=0.5$ & $\alpha=1$ \\
\hline \multirow[t]{2}{*}{3} & 3.6570 & 3.6570 & 2.8736 & 2.1630 & 1 & 2.3096 & 1.7889 & 2.2232 & 1.4942 \\
\hline & & & & & -1 & 5.5066 & 3.1696 & 6.2195 & 4.4246 \\
\hline \multirow[t]{2}{*}{4} & 5.1355 & 5.1355 & 3.7963 & 2.8197 & 1 & 3.0854 & 2.3558 & 2.8862 & 1.9076 \\
\hline & & & & & -1 & 6.6168 & 7.7715 & 5.5641 & 5.1806 \\
\hline \multirow[t]{2}{*}{5} & 5.2143 & 5.2143 & 4.7793 & 3.7483 & 1 & 1.7104 & 2.1492 & 2.3176 & 2.1088 \\
\hline & & & & & -1 & 2.3269 & 4.5273 & 5.2070 & 4.0432 \\
\hline
\end{tabular}




\section{Conclusions}

We present Bayesian estimation based on SRS and MRSSU. The Weibull distribution is used as an application example to illustrate our results. We compute bias, MSE and relative efficiency of the derived Bayesian estimates and then make a comparison between SRS and MRSSU. Our observations of the results are stated in the following points:

1) From Table 1 and Table 2, first, we found that the Bayesian estimates of $\alpha$ are all biased. Next, we found that the Bayesian estimates based on Jeffreys prior are less biased than gamma prior. Also, we observed that the Bayesian estimates based on MRSSU are considerably less biased than SRS.

2) From Table 3 and Table 4, it is observed that the mean squared error of all estimates decreases when sample size $m$ increases. Next, we observed that the Bayesian estimates based on MRSSU have a much smaller mean squared error than the corresponding Bayesian estimates based on SRS in all cases considered.

3) From Table 5, we observe that the relative efficiency of the Bayesian estimator based on MRSSU w.r.t. SRS Bayesian estimators are greater than 1 and increases with $m$. Also, decreases in Linex function as $m$ increases for $m=5$.

Therefore, we conclude that the Bayesian estimates based on maximum ranked set sampling with unequal samples are more efficient than the corresponding Bayesian estimates of simple random sampling.

Finally, we conclude that the results of the simulation experiment showed that the Bayesian estimates based on maximum ranked set sampling with unequal samples are more efficient, when compared with the Bayesian estimates of simple random sampling.

\section{Acknowledgements}

The authors would like to thank the referees for their helpful comments that have led to an improved paper.

\section{References}

[1] McIntyre, G.A. (1952) A Method for Unbiased Selective Sampling Using Ranked Sets. Australian Journal of Agricultural Research, 3, 385-390. https://doi.org/10.1071/AR9520385

[2] Takahasi, K. and Wakimoto, K. (1968) On Unbiased Estimates of the Population Mean Based on the Sample Stratified by Means of Ordering. Annals of the Institute of Statistical Mathematics, 20, 1-31. https://doi.org/10.1007/BF02911622

[3] Dell, T.R. and Clutter, J.L. (1972) Ranked Set Sampling Theory with Order Statistics Background. Biometrics, 28, 545-555. https://doi.org/10.2307/2556166

[4] Shaibu, A.B. and Muttlak, H.A. (2004) Estimating the Parameters of the Normal, Exponential and Gamma Distributions Using Median and Extreme Ranked Set Samples. Statistics, 1, 75-98.

[5] Al-Omari, A.I., Jaber, K.H. and Al-Omari, A. (2008) Modified Ratio-Type Estimators of the Mean Using Extreme Ranked Set Sampling. Journal of Mathematics and Statistics, 4, 150-155. https://doi.org/10.3844/jmssp.2008.150.155 
[6] Islam, T., Shaibur, M.R. and Hossain, S.S. (2009) Effectivity of Modified Maximum Likelihood Estimators Using Selected Ranked Set Sampling Data. Austrian Journal of Statistics, 38, 109-120.

[7] Ibrahim, K. and Syam, M. (2010) Estimating the Population Mean Using Stratified Median Ranked Set Sampling. Applied Mathematical Sciences, 4, 2341-2354.

[8] Varian, H.R. (1975) A Bayesian Approach to Real Estate Assessment. North Holland, Amsterdam, 195-208.

[9] Zellner, A. (1986) Bayesian Estimation and Prediction Using Asymmetric Loss Functions. Journal of the American Statistical Association, 81, 446-451. https://doi.org/10.1080/01621459.1986.10478289

[10] Al-Saleh, M.F. and Muttlak, H.A. (1998) A Note in Bayesian Estimation Using Ranked Set Sampling. Pakistan Journal of Statistics, 14, 49-56.

[11] Ahmed (2007) Bayesian Estimation of the Logormal Distrbution Mean Using Ranked SET Sampling. Basrah Journal of Science, 25, 101-112.

[12] Sadek, A., Sultan, K.S. and Balakrishnan, N. (2009) Bayesian Estimation Based on Ranked Set Sampling Using Asymmetric Loss Function. Bulletin of the Malaysian Mathematical Sciences Society, 38, 707-718.

[13] Sadek, A. and Alharbi, F. (2014) Weibull-Bayesian Analysis Based on Ranked Set Sampling. International Journal of Advanced Statistics and Probability, 2, 114-123. http://dx.doi.org/10.14419/ijasp.v2i2.3373

[14] Al-Hadhrami, S.A. and Al-Omari, A.I. (2009) Bayesian Inference on the Variance of Normal Distribution Using Moving Extremes Ranked Set Sampling. Journal of Modern Applied Statistical Methods, 8, 273-281.

[15] Hassan, A.S. (2013) Maximum Likelihood and Bayes Estimators of the Unknown Parameters for Exponentiated Exponential Distribution Using Ranked Set Sampling. International Journal of Engineering Research and Applications, 3, 720-725.

[16] Mohammadi, M.Y. and Pazira, H. (2010) Classical and Bayesian Estimations on the Generalized Exponential Distribution Using Censored Data. International Journal of Mathematical Analysis, 4, 1417-1431.

[17] Ghafoori, S., Habibi Rad, A. and Doostparast, M. (2011) Bayesian Two-Sample Prediction with Type-II Censored Data for Some Lifetime Models. JIRSS, 10, 63-86.

[18] Al-Hadhrami, S.A. and Al-Omari, A.I. (2012) Bayes Estimation of the Mean of Normal Distribution Using Moving Extreme Ranked Set Sampling. Pakistan Journal of Statistics and Operation Research, VIII, 21-30.

[19] Mohie El-Din, M.M., Kotb, M.S. and Newer, H.A. (2015) Bayesian Estimation and Prediction for Pareto Distribution Based on Ranked Set Sampling. Journal of Statistics Applcations and Probability, 4, 211-221.

[20] Biradar, B.S. and Sanotsha, C.D. (2014) Estimation of the Mean of the Exponential Distribution Using Maximum Ranked Set Sampling with Unequal Samples. Open journal of Statistics, Scientific Research, 4, 641-649.

[21] Berger, J.O. (1985) Statistical Decision Theory and Bayesian Analysis. Springer-Verlag, New York. https://doi.org/10.1007/978-1-4757-4286-2 
Submit or recommend next manuscript to SCIRP and we will provide best service for you:

Accepting pre-submission inquiries through Email, Facebook, LinkedIn, Twitter, etc. A wide selection of journals (inclusive of 9 subjects, more than 200 journals)

Providing 24-hour high-quality service

User-friendly online submission system

Fair and swift peer-review system

Efficient typesetting and proofreading procedure

Display of the result of downloads and visits, as well as the number of cited articles

Maximum dissemination of your research work

Submit your manuscript at: http://papersubmission.scirp.org/

Or contact ojs@scirp.org 